\title{
Vulvar Dermatoses in Libyan Adult Patients
}

\section{Suleman Elfaituri S*}

Dermatology department, medical faculty, Benghazi University, Libya

*Corresponding author: Safa Suleman Elfaituri, Associated Professor, Dermatology department, medical faculty, Benghazi university, Libya, Email:

\section{Research Article}

Volume 4 Issue 1

Received Date: December 18, 2018

Published Date: January 17, 2019

DOI: $10.23880 /$ cdoaj- 16000168 selfaitoury@yahoo.co.uk

\section{Abstract}

Vulvar dermatoses are common problems in women and they are associated with significant morbidity. Their frequency and importance are often underestimated. In a prospective study we evaluated 200 adult females presenting vulvar symptoms and determined the spectrum and frequency of vulvar dermatoses in a Libyan population. Mean age was 38 years, $90 \%$ was married and 5\% was pregnant ladies. The commonest condition was vulvovaginal candidiasis (22\%) followed by genital warts (19\%), bacterial infections (15\%) contact dermatitis (11\%), lichen simplex chronicus (8\%) and other conditions. This study provided important data on the spectrum of vulvar dermatoses in Libyan adult patients and to the best of our knowledge it is the first study carried out on vulvar dermatoses in Libya.

Keywords: Vulvar dermatoses; Vulvovaginal candidiasis; Women; Libya

\section{Introduction}

Vulvar dermatoses may be associated with mental distress and guilt feelings in affected patients. They have been classified by the International Society for the Study of Vulvovaginal Disease (ISSVD) to venereal and nonvenereal vulvar dermatoses. Venereal diseases include herpes and syphilis and non-venereal dermatoses include inflammatory cutaneous disorders as psoriasis, lichen planus and lichen sclerosus, autoimmune (vitiligo), multisystem diseases as Behcet's disease and Crohn's disease, exogenous as contact dermatitis and fixed drug eruption, and neoplasms as extramammary Paget disease [1].

Although vulvar disease is common, relatively few epidemiological studies are available from undeveloped countries. Moreover, no information exists from Benghazi, Libya. The aim of this study was to determine the spectrum and frequency of vulvar dermatoses among patients of a dermatology outpatient clinic in BenghaziLibya.

\section{Materials \& Methods}

A prospective study was performed on adult females with genital complaints attended at the dermatology outpatient clinic in Jumhoria hospital and IbnSina polyclinic, Benghazi-Libya for a period of three years from 2012 to 2014. A detailed history including age, menstrual, marital and pregnancy status, sexual exposure, main complaints (itching, pain, dyspareunia) was recorded. Associated medical or skin disease as well as drug history was obtained. Examination of external genitalia was carried out and complete skin examination was done including scalp, mouth and nails in selected cases. Relevant investigations including screening for sexually transmitted infections and skin biopsy were performed, whenever indicated.

The vulvar dermatoses were categorized into infectious dermatoses (bacterial, fungal and viral), eczematous dermatitis, squamous papular dermatoses, bullous dermatoses, drug rashes, neoplasms and miscellaneous. 


\section{Clinical Dermatology Open Access Journal}

The data were statistically analyzed using software SPSS, Version 13.0 (IBM Corp, Armonk, NY).

\section{Results}

A total of two hundred women were included. Majority of patients was married (90\%). The age of patients ranged from 20 years to 92 years with a mean age of 38 . Ten percent was postmenopausal whereas $90 \%$ was in reproductive age and $5 \%$ was pregnant.

The commonest condition was infection (60\%); fungal infections were the commonest; including vulvovaginal candidiasis $(22 \%)$ and tinea cruris $(2 \%)$.

Genital wart (19\%), herpes simplex (1\%), herpes zoster $(0.5 \%)$ and molluscum contagiosum $(0.5 \%)$ were the main viral infections reported in this study.

Bacterial infections included folliculitis and boils (13\%) ecthyma and cellulitis (1\%) Contact dermatitis (11\%) and lichen simplex chronicus (8\%) were the main eczematous dermatitis. Papulosquamous disease included psoriasis (4\%), lichen planus (3\%) and lichen sclerosus (2\%). Bullous dermatoses involving vulva (4\%) were gestational pemphigoid, pemphigus and linear Ig A dermatosis. Drug rashes was reported in 2\%. Other conditions included lymphedema, histiocytosis, Crohn's, Behcet's, skin tags, sebaceous hyperplasia, angioedema and vitiligo. Figures from 1 to 13 show variable vulvar dermatoses.

\section{Discussions}

Vulvar dermatoses affect many women. The warm and moist environment of the vulva with its keratinized and non-keratinized portions and predisposition to friction, make it at a particular risk for the development of different dermatoses.

Vulvar dermatoses may cause itching, pain and dyspareunia and can provoke emotional distress and anxiety. They may severely affect the woman's quality of life by impacting body image and sexual function [2-5]. Genital diseases are likely underdiagnosed due to embarrassment and reluctance to visit a health care provider [6].

The most common vulvar dermatoses noted in our study was infections, in concordance with Bauer, et al. finding [7]. Candidiasis is a common vulvovaginal infection, most commonly caused by C. albicans,. It may or may not present with a white, curd-like discharge [8]. It was the commonest vulvar problem in concordance with Iraqi report [9] but in contrast to Singh, et al. results where lichen sclerosis was the commonest cause of symptomatic vulval disease [10].

The increased frequency of candidiasis in this study may reflect the wrong medical practice and overuse of antibiotics to all patients complaining of dysuria without investigations. Secondary candidiasis may occur in patient treated with topical steroids [11].

Genital warts are extremely common; they are the most common sexually transmitted infection and are important health problem because of their association with cervical cancer [12]. It was the second most common infection in our study. Although herpes simplex virus infection is a common cause of recurrent vulvar pain as well as it is the most common cause of genital ulcers, it constituted only $1 \%$ of cases in our study. Herpes zoster is not common in the genital area [8], only one case was reported [13-15].

Folliculitis was a common vulvar problem, constituted $13 \%$ of cases; frequent shaving, occlusive clothing, exposure to hot humid temperatures, diabetes mellitus, and obesity is predisposing factors [9].

Different types of dermatitis can affect the vulvar area; irritant contact dermatitis is the commonest type of eczema to affect the vulva. The vulvar epithelium is less efficient as a barrier than skin elsewhere. Frequent washing, soaps, home remedies, fragrances, and wearing tight clothing, or pads can exacerbate the dermatitis. Genital lichen simplex chronicus may be primary in atopic or superimposed on itchy vulvar dermatoses, as eczema, lichen sclerosus or candidiasis [3]. Vulvar dermatitis in our study was observed in 19\% near to Ball, et al. findings [16].

Vulvar psoriasis is a manifestation of flexural psoriasis and it is usually intensely provoked in this area because of friction, sweating, heat and scratching [14]. In this study psoriasis was an uncommon vulvar dermatosis (4\%), similar to that of other vulvar clinics $(1.8-5 \%)[9,14]$.

Vulvar lichen planus may presents as classical, hypertrophic or erosive with or without a lacy white border [8]. Vulvar erosive lichen planus usually present with pain and leads to considerable scarring and loss of anatomy. 
Lichen sclerosus is chronic inflammatory dermatoses. Sexual dysfunction is common with entry dyspareunia because of erosions, scarring and introital narrowing. Squamous cell carcinoma (SCC) can occur in up to $5 \%$ of women with untreated disease $[8,15]$.

Our finding was in contrast to studies quoted in western literature where lichen sclerosus et atrophicus was the most frequently observed vulvar dermatoses (39\%), and lichen planus was the third most common dermatois $(11.5 \%)[16,17]$.

Lymphedema is swelling attributed to accumulation of lymph in tissue. It is associated with inadequate lymphatic drainage which may be primary or secondary to trauma as surgery, inflammation, and malignancy [14]. We had one case of lymphedema.

Acrochordon is a pedunculated fibroepithelial polyp commonly seen in intertriginous areas. It may be seen on labia majora and labia minora. It can be single or multiple with size varying from a few millimeters to one $\mathrm{cm}$ [18]. We observed acrochordon in two obese women, and giant tag in one patient.

The most common vulvar cancer is squamous cell carcinoma, and it is a complication in lichen sclerosus and lichen planus. Vulvar Paget's disease is very rare malignancy originating in vulvar apocrine gland or as a manifestation of adjacent primary anal, rectal or bladder adenocarcinoma. It appears clinically as chronic eczematous lesions [19]. Vulvar cancer should be considered for any persistent, fissured, eroded or ulcerated vulvar condition [8]. No cases of neoplasia were reported in our study.

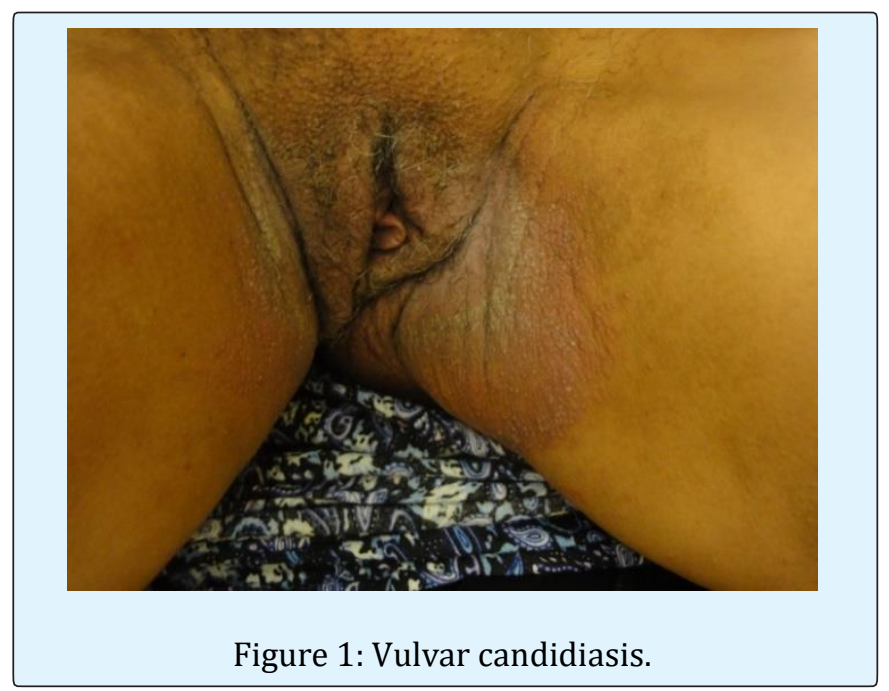

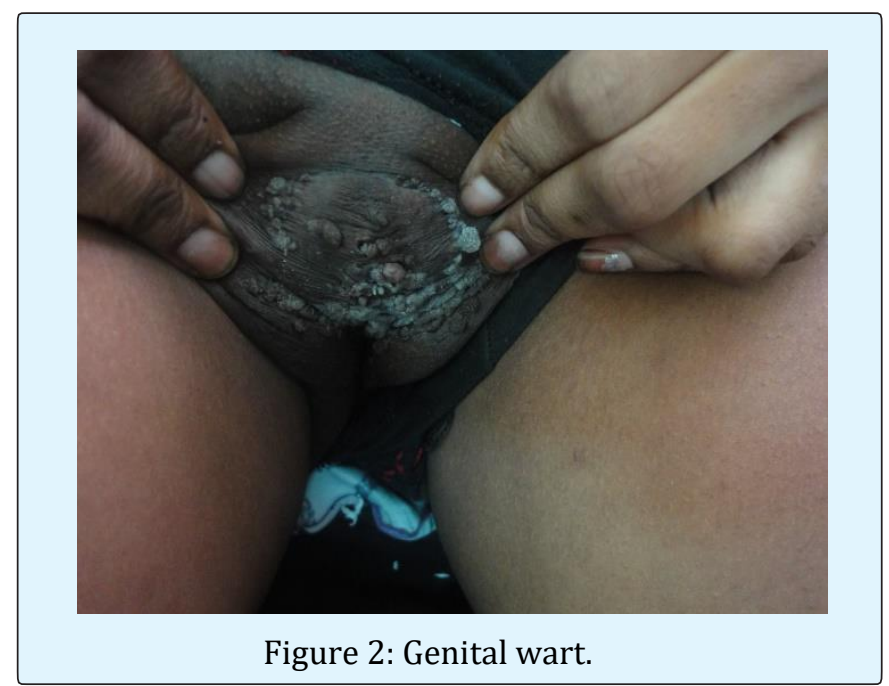
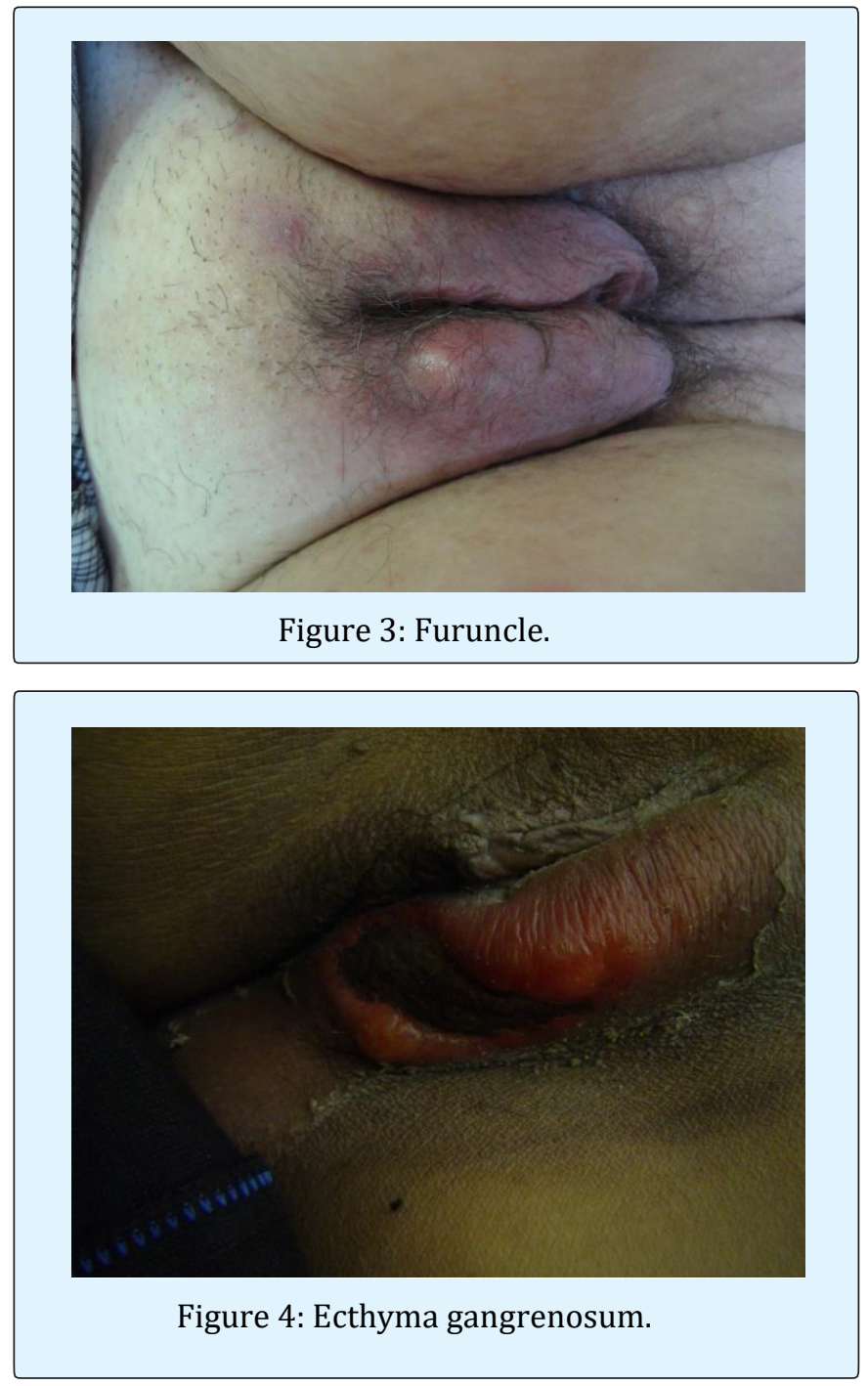

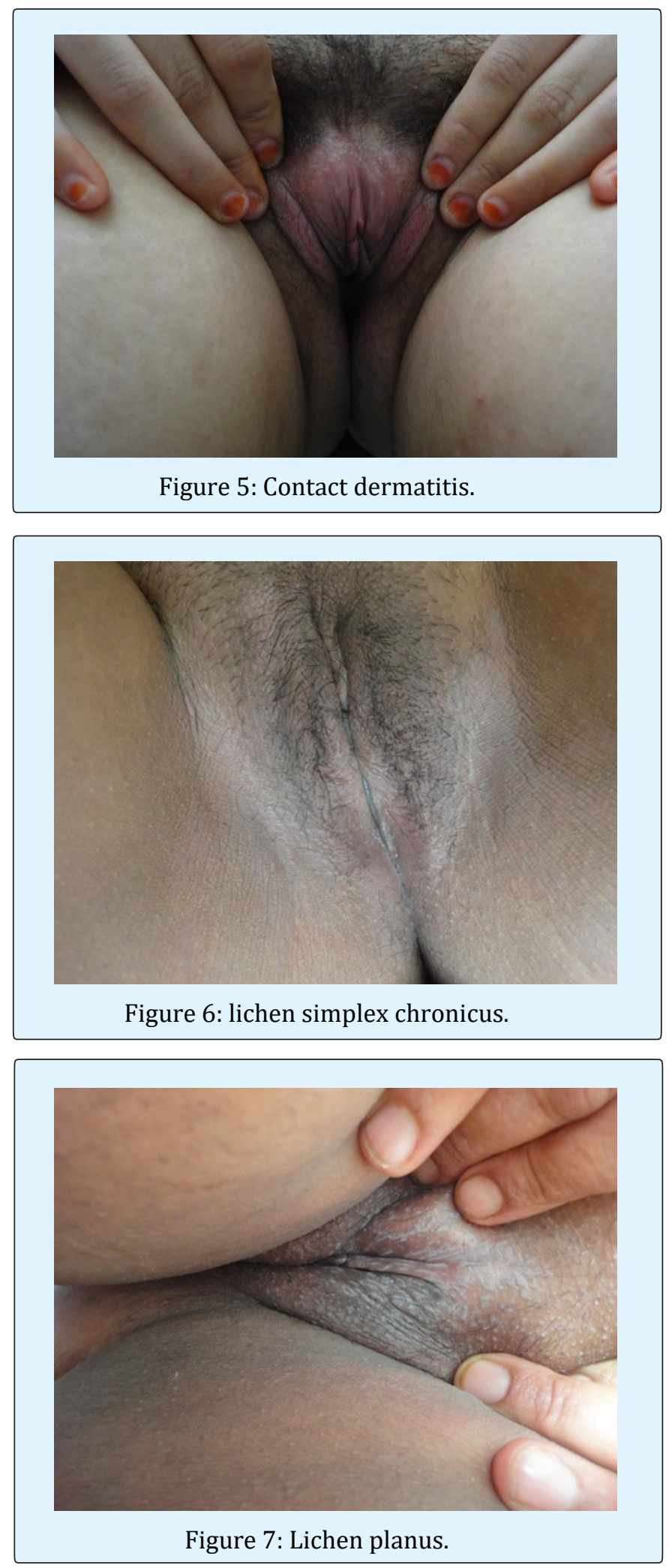
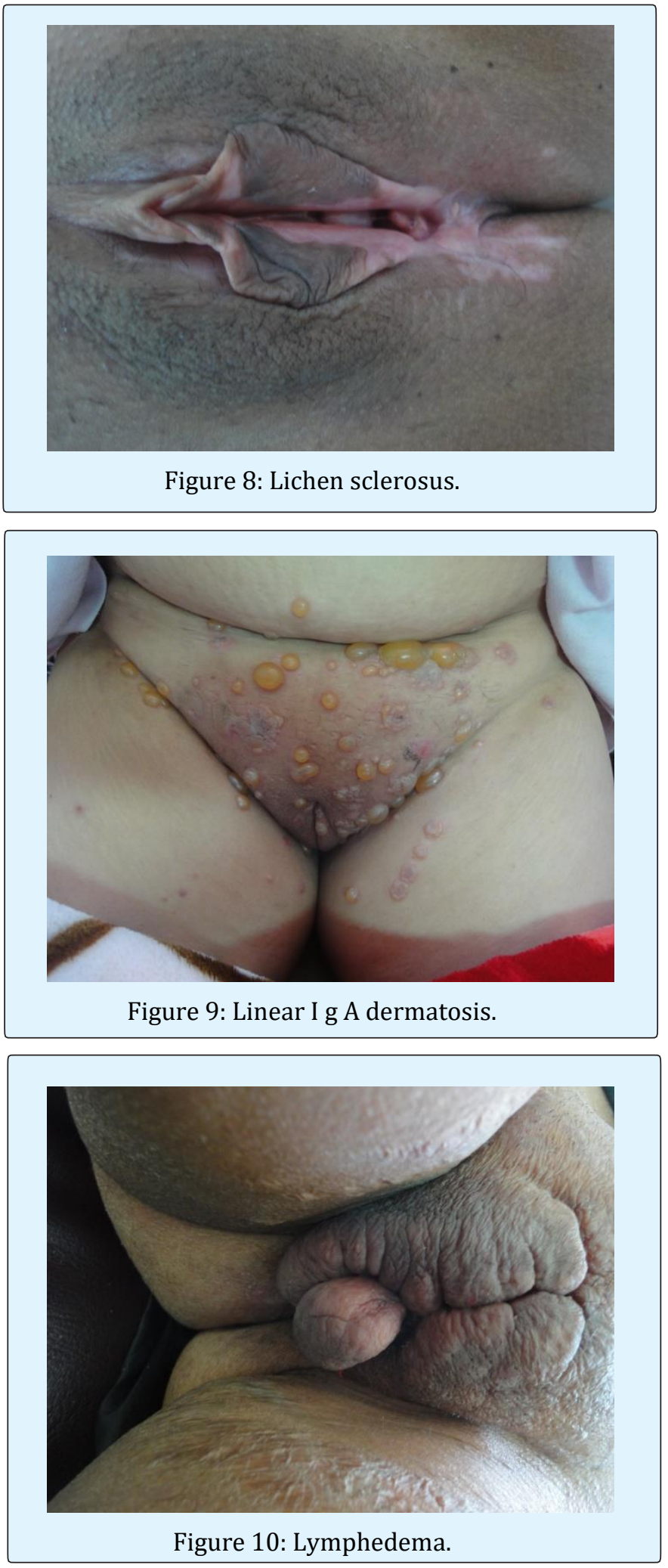

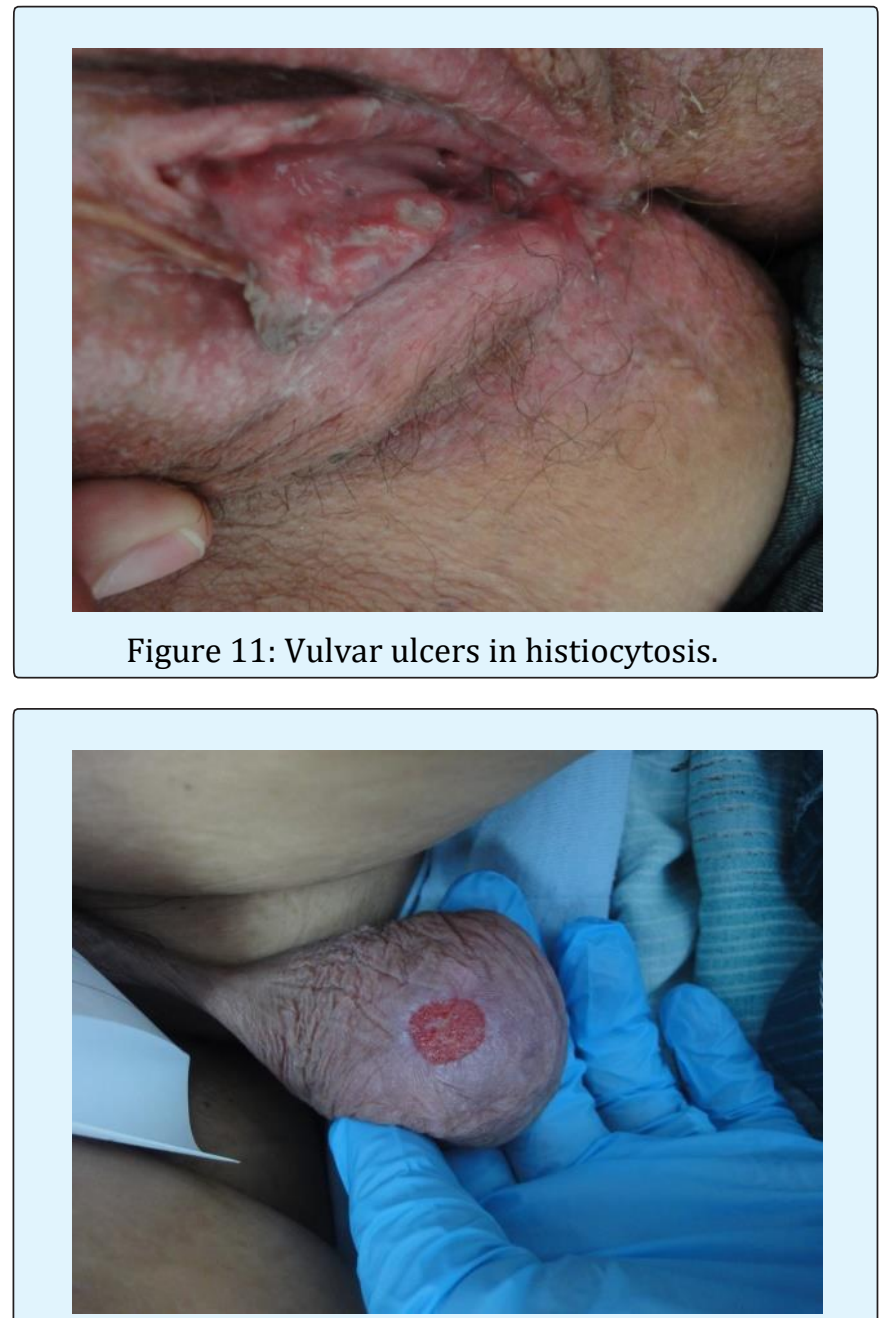

Figure 12: Giant skin tag with ulceration.

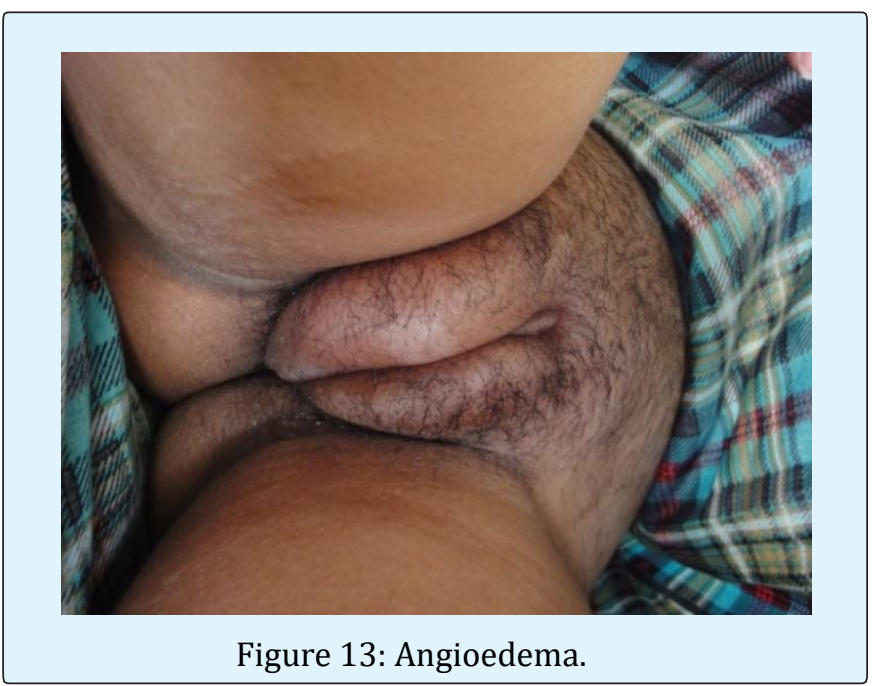

\section{Conclusions}

This study provides important data on the spectrum of vulvar diseases in Libyan adult patients. The most common vulvar diseases observed in this study were candidiasis and genital warts. Recognition and treatment of vulvar disease is important for symptom relief, sexual function and prevention of progression to vulvar scarring and cancer.

To the best of our knowledge this is the first study carried out on vulvar dermatoses in Libya.

\section{References}

1. Lynch PJ, Moyal-Barracco M, Bogliatto F, Micheletti L, Scurry J (2007) 2006 ISSVDClassification of vulvar dermatoses: pathologic subsets and their clinical correlates. J Reprod Med 52(1): 3-9.

2. Ferensowicz C (2013) Vulvar dermatoses: an NP's approach to benign skin conditions "down there".

3. Burrows LJ, Shaw HA, Goldstein AT (2008) The vulvar dermatoses. J Sex Med 5(2): 276-283.

4. Thorstensen KA, Birenbaum DL (2012) Recognition and management of vulvar dermatologic conditions: lichen sclerosus, lichen planus, and lichen simplex chronicus. J Midwifery Womens Health 57(3): 260275.

5. Foster DC (2002) Vulvar disease. Obstet Gynecol. 100(1): 145-163.

6. Guerrero A, Venkatesan A (2015) Inflammatory Vulvar Dermatoses. Clin Obstet Gynecol 58(3): 464475.

7. Bauer A, Greif C, Vollandt R, Merker A, Elsner P (1999) Vulval diseases need an interdisciplinary approach. Dermatology 199(3): 223-226.

8. Danby CS, Margesson LJ (2010) Approach to the diagnosis and treatment of vulvar pain. Dermatol Ther 23(5): 485-504

9. Al-Yasin ZT (2009) The frequency of symptomatic vulval disease: a gynaecological's perspective. The medical journal of basrah university. Iraq MJBU 27(2): 104-107. 
10. Singh N, Thappa DM, Jaisankar TJ, Habeebullah S (2008) Pattern of non-venereal dermatoses of female external genitalia in South India. Dermatol Online J 14(1): 1 .

11. Dennerstein G (2018) Re: The management of vulval itching caused by benign vulval dermatoses, The Obstetrician \& Gynaecologist 20(2): 136.

12. Wiley DJ, Douglas J, Beutner K, Cox T, Fife K, et al. (2002) External genital warts: diagnosis, treatment, and prevention. Clinical Infectious Diseases, Clin Infect Dis 35(2): S210-S224.

13. Van der Meijden WI, Boffa MJ, Ter Harmsel WA, Kirtschig G, Lewis FM, et al. (2017) 2016 European guideline for the management of vulval conditions. JEADV 31(6): 925-941.

14. Fischer G, Bradford J (2010) The Vulva: A Clinician's Practical Handbook. Family Planning New South Wales.
15. Puri N, Puri A (2012) A study on non venereal genital dermatoses in north india. Our dermatol online 3(4): 304-307.

16. Ball SB, Wojnarowska F (1998) Vulvar dermatoses: lichen sclerosus, lichen planus, and vulval dermatitis/lichen simplex chronicus. Semin Cutan Med Surg 17(3): 182-188.

17. Cheung ST, Gach JE, Lewis FM (2006) A retrospective study of the referral patterns to a vulval clinic: highlighting educational needs in this subspecialty. Journal of Obstetrics and Gynaecology 26(5): 435437.

18. Dey M, Kumar R, Sriram R (2014) Giant acrochordon of vulva. Med Sci 3(2): 1299-1304.

19. Anton C, Luiz AV, Carvalho FM, Baracat EC, Carvalho JP (2011) Clinical treatment of vulvar Paget's disease: a case report. Clinics (Sao Paulo) 66(6): 1109-1111. 